\title{
A review of telavancin in the treatment of complicated skin and skin structure infections (cSSSI)
}

\author{
Lala M Dunbar' \\ Derek M Tang ${ }^{2}$ \\ Robert M Manausa' \\ 'Louisiana State University Health \\ Sciences Center, New Orleans, USA; \\ ${ }^{2}$ Tulane University School of Medicine, \\ USA
}

\begin{abstract}
Telavancin is a novel antibiotic being investigated for the treatment of serious infections caused by Gram-positive bacteria, including complicated skin and skin structure infections (cSSSI) and pneumonia. This once-daily intravenous lipoglycopeptide exerts rapid bactericidal activity via a dual mechanism of action. It is intended for use to combat infections caused by Staphylococcus aureus and other Gram-positive bacteria, including methicillinresistant and vancomycin-intermediate strains of $S$. aureus (MRSA and VISA, respectively). Vancomycin is the current gold standard in treating serious infections caused by Gram-positive bacteria, especially MRSA. In recent clinical trials, telavancin has shown excellent efficacy in phase II and III multinational, randomized, double-blinded studies of cSSSI. In the phase II FAST 2 study, which compared telavancin $10 \mathrm{mg} / \mathrm{kg}$ intravenously q $24 \mathrm{~h}$ vs standard therapy (an antistaphylococcal penicillin at $2 \mathrm{~g}$ IV q $6 \mathrm{~h}$ or vancomycin 1 gm IV q $12 \mathrm{~h}$ ), the clinical success rate in the telavancin-treated group was $96 \%$ vs $94 \%$ in the standard therapy group. In two identical phase III trials comparing telavancin versus vancomycin at the doses of the FAST 2 study for cSSSI, the clinical cure rates were $88.3 \%$ and $87.1 \%$, respectively. Two additional phase III clinical trials investigating telavancin for use in hospital-acquired pneumonia, caused by Gram-positive bacteria are currently ongoing. Telavancin is currently under regulatory review in both the United States and Europe for the indication of treatment of cSSSI.
\end{abstract}

Keywords: telavancin, vancomycin, MRSA

\section{Introduction}

In 1928, Sir Alexander Fleming's discovery and isolation of penicillin from the penicillium mold marked the beginning of modern antibiotics. By the early 1940s, Pfizer had developed a deep-tank fermentation method to produce mass quantities of penicillin for pharmaceutical use (Pfizer 2007). This "miracle drug" has since become the most widely used antibiotic to date and it is active against many Gram-positive bacteria. Universal sensitivity of common Gram-positive bacteria to penicillin was, however short lived. By 1945, resistance to penicillin mediated by $\beta$-lactamase production was reported in isolates of Staphylococcus aureus, one of the most common and virulent Gram-positive bacteria encountered clinically (Spink and Ferris 1945). In 1950, 40\% of hospital S. aureus isolates were penicillin-resistant, and by 1960 , this proportion had increased to $80 \%$ (Chambers 2001). Consequently, penicillin is no longer a consideration in the empiric management of infections likely to be caused by $S$. aureus. This pathogen causes serious invasive infections, such as community acquired and nosocomial pneumonia, endocarditis, soft tissue infections, and bacteremia (Drew 2007).

Initial S. aureus resistance was mediated by bacterial production of $\beta$-lactamase or penicillinase enzymes that could cleave the $\beta$-lactam ring structure of the antibiotic, rendering it ineffective. As a result, $\beta$-lactam antibiotics, such as methicillin, cloxacillin, and oxacillin, were developed with modification of the chemical structure that protected 
the $\beta$-lactam ring from cleavage by the $\beta$-lactamase enzymes. These antibiotics soon became the mainstay of empiric treatment for infections thought to be caused by $S$. aureus. Introduced in 1959, methicillin was the first antibiotic of the class to be used, but by 1961, the first case of methicillinresistant $S$. aureus (MRSA) was documented during routine screening of hospital isolates at a reference laboratory in England (Jevons 1961).

To compound matters, a growing medical concern has been the emergence of multidrug-resistant strains among isolates of S. aureus (Goossens 2006). In the 1990s, hospitalacquired MRSA (HA-MRSA), which had generally remained uncommon, suddenly surged in prevalence and is now endemic in this setting (Johnson et al 2001). In 2006, results of the Surveillance Network USA showed that nearly $60 \%$ of hospital-derived $S$. aureus isolates were MRSA (Styers et al 2006). One of the most disturbing features of HA-MRSA is its multi-drug resistance, including resistance to all the $\beta$-lactam antibiotics, penems and carbapenems. Resistance to multiple other antibiotic classes including aminoglycosides, macrolides, and tetracyclines, as well as the antibiotic trimethoprim is common through plasmid mediated transfer of resistance (Swartz 1994). Point mutations on DNA gyrase and topoisomerase IV, and hyperexpression of efflux pump proteins accounted for the rapid resistance of HA-MRSA to flouroquinolones shortly after their widespread use (Hershow et al 1998; Hooper 1999). In the early 2000s, a new strain of community-acquired MRSA (CA-MRSA) was identified in infections acquired in the community setting in patients who had no identifiable risk factors for the HA strain. Resistance in CA-MRSA is also mediated through acquisition and expression of PBP-2a, which provides resistance to methicillin, but unlike the hospital-acquired strain, CA-MRSA presently remains highly sensitive to clindamycin, rifampin, and trimethoprim-sulfamethoxazole, and the tetracyclines (Moran et al 2006). CA-MRSA differs from the early HA strains (now broadened to healthcare-associated (HCA)) by being more strongly associated with a virulence factor, the Panton-Valentine Leukocidin (PVL) toxin, which seems to be responsible for its invasiveness and necrotizing properties (Davis et al 2007). Failure to inhibit production of PVL may play a significant role in outcome of serious necrotizing infections and will likely impact the choice of antibiotics used. Findings from a prospective surveillance study conducted in 11 emergency departments across the US has helped to characterize the etiology of complicated skin and skin structure infections (cSSSIs) (Moran et al 2006). S. aureus was isolated from the infection site in 320 of 422 patients $(76 \%)$. Furthermore, the overall prevalence of MRSA was 59\% nearly all of which had pulsed-field types characteristic of CA-MRSA. Genes encoding $p v l$ were contained within $98 \%$ of MRSA isolates and $40 \%$ of MSSA isolates. Other bacterial pathogens such as Streptococcus spp. (7\%), coagulase-negative staphylococci (3\%) and Proteus mirabilis $(1 \%)$ were identified as a small but significant cause of cSSSI in this study. For this reason, these purulent infections were commonly treated with non- $\beta$-lactam antibiotics, such as clindamycin and trimethoprim-sulfamethoxazole (Moran et al 2006). Results from another well conducted prospective epidemiologic study found lower rates of PVL expression among $S$. aureus, although $54 \%$ of CA-MRSA isolates $(n=102)$ and $10 \%$ of MSSA isolates $(n=102)$ still carried $p v l$ genes (Davis et al 2007). Overall, these microbiological data highlight important knowledge deficits and/or the dynamic environment surrounding the epidemiology and causes of cSSSIs.

In an article from the Infectious Disease Society of America written in 2006 (Talbot et al 2006) entitled "Bad Bugs, Need Drugs", the dilemma of treating emerging resistant organisms and the dearth of new antibiotics in development was highlighted. In response to this need, several new antibiotics have recently been approved and are currently in use to treat serious infections caused by resistant strains of S. aureus. This list includes: linezolid, tigecycline, and daptomycin. There are a number of other agents in various stages of development and regulatory review such as the lipoglycopeptides telavancin, oritavancin, and dalbavancin, as well as agents from traditional antimicrobial drug classes (eg, the cephalosporins, ceftobiprole, and ceftaroline, and the trimethoprim congener iclaprim).

Currently, glycopeptide antibiotics such as vancomycin and teicoplanin are the gold standard for the treatment of serious invasive infections due to MRSA (although teicoplanin is not approved for use in the United States) (Van Bambeke 2004; Rice 2006). Vancomycin, available since 1950 , is presently considered to be the preferred treatment for invasive infections caused by MRSA. This is in part due to its favorable safety profile as well as extensive published studies documenting its utility in the treatment of serious invasive infections (Drew 2007).

The utility of vancomycin is, however, compromised by several important factors. These include: (1) poor penetration of the antibiotic into infected tissues in the lungs, brain, and meninges, which has been associated with unfavorable treatment outcomes in serious infections such as pneumonia, endocarditis and meningitis (Levine 2006; Stevens 2006; 
Drew 2007); (2) an adverse effect of biofilms produced by several $S$. aureus strains on vancomycin's microbiologic activity (Nishimura et al 2006; Sakoulas et al 2006; Drew 2007); and (3) emergence of resistance to vancomycin in enterococci and staphylococci (Moellering 2006). The first vancomycin-intermediate $S$. aureus (VISA) isolate was reported in Japan in 1996 (Hiramatsu et al 1997). Subsequently 2 cases of vancomycin-resistant $S$. aureus (VRSA) were first reported in the United States in 2002 (CDC 2002a, b). It is increasingly recognized that many $S$. aureus cultures are 'heteroresistant' (hVISA) containing a subpopulation of resistant organisms which survive antibiotic therapy (Maor et al 2007). Overall there has been an increase in the MICs of vancomycin against MRSA, associated with poorer outcomes, which has prompted the Clinical and Laboratory Standards Institute (CLSI) to lower vancomycin's breakpoint from $\leq 4 \mu \mathrm{g} / \mathrm{mL}$ to $\leq 2 \mu \mathrm{g} / \mathrm{mL}$ for susceptible strains, from $8-16 \mu \mathrm{g} / \mathrm{mL}$ to $4-8 \mu \mathrm{g} / \mathrm{mL}$ for intermediatelysusceptible strains, and from $\geq 32 \mu \mathrm{g} / \mathrm{mL}$ to $\geq 16 \mu \mathrm{g} / \mathrm{mL}$ for resistant strains (CLSI 2007).

The emergence and prevalence of multidrug-resistant Gram-positive pathogens, as well as their increasing contribution to nosocomial and serious community acquired infections, has prompted the development of several new antimicrobials. This review focuses on telavancin, and gives a general summary of the most recent published data.

\section{Chemistry}

Telavancin is a lipoglycopeptide, structurally similar to vancomycin (Figure 1). It has the same glycopeptide core as vancomycin, but has an added lipophilic side chain (decylaminoethyl) and a negatively charged group (phosphonomethyl aminomethyl). The lipophilic side chain has been hypothesized to enhance telavancin's membraneanchoring properties leading to enhanced affinity for lipid II (Beauregard et al 1995; Leadbetter et al 2004; Laohavaleeson et al 2007). The polar moiety compensates for the prolonging effect of the lipophilic tail on elimination half-life by endowing the molecule with a partition coefficient that ensures desirable absorption, distribution, metabolism, and renal excretion properties (Judice and Pace 2003; Leadbetter et al 2004; Laohavaleeson et al 2007).

\section{Mechanism of action}

Telavancin is a concentration-dependent, rapidly bactericidal antibiotic which has potent activity against clinically important Gram-positive pathogens such as staphylococci (including MRSA, hVISA, and VISA strains) and streptococci, (including penicillin-resistant Staphylococcus pneumoniae PRSP) as well as Gram-positive anaerobic and fastidious aerobic bacteria. Telavancin activity is mediated by multiple modes of action. In a mechanism shared with vancomycin, telavancin inhibits bacterial cell wall synthesis through tight binding of the aglycone core structure to D-alanineD-alanine-containing peptidoglycan precursor, lipid II, and nascent noncrosslinked peptidoglycan intermediates, thus inhibiting late stages of cell wall biosynthesis (Higgins et al 2005). Telavancin is approximately 10 -fold more potent than vancomycin in the inhibition of peptidoglycan synthesis. Additionally, in mechanisms not shared with vancomycin,
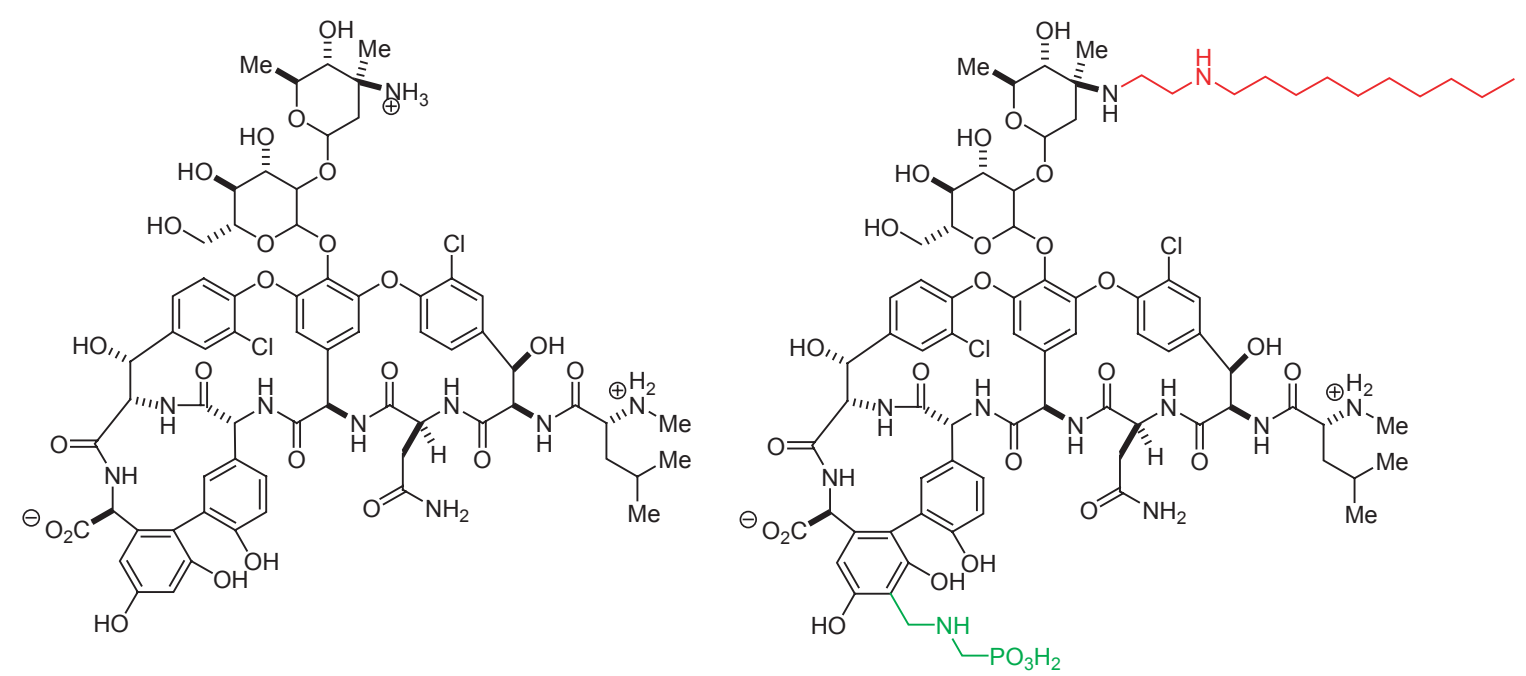

Figure I Chemical structures of vancomycin (left) and telavancin (right). Telavancin, structurally related to vancomycin, possesses a lipophilic side chain (decylaminoethyl, highlighted in red) and a negatively charged group (phosphonomethyl aminomethyl, highlighted in green). 
Table I In vitro activities of telavancin and comparators against key Gram-positive bacteria. Data taken from surveillance studies evaluating clinical isolates

\begin{tabular}{|c|c|c|c|c|}
\hline $\begin{array}{l}\text { Antibacterial/organism } \\
\text { (no. of isolates) }^{\mathrm{a}}\end{array}$ & MIC range $(\mu \mathrm{g} / \mathrm{ml})$ & $\begin{array}{l}\text { MIC }_{90} \text { range } \\
(\mu \mathrm{g} / \mathrm{ml})\end{array}$ & $\%$ Susceptible ${ }^{b}$ & References \\
\hline \multicolumn{5}{|c|}{ Staphylococcus aureus: methicillin susceptible $(n=25$ I5) } \\
\hline Telavancin & $\leq 0.015-1$ & $0.25-0.5$ & NA & \multirow{4}{*}{$\begin{array}{l}\text { (Draghi et al 2006; Sahm } \\
\text { et al 2006; Jansen et al } \\
2007 \text { ) }\end{array}$} \\
\hline Vancomycin & $\leq 0.25-2$ & 1 & 100 & \\
\hline Linezolid & $\leq 0.5->4$ & 2 & $99.8-100$ & \\
\hline Daptomycin & $\leq 0.03->1$ & 0.5 & 99.9 & \\
\hline \multicolumn{5}{|c|}{ Staphylococcus aureus: methicillin resistant $(\mathrm{n}=$ I 669) } \\
\hline Telavancin & $0.06-1$ & $0.25-0.5$ & NA & \multirow{4}{*}{$\begin{array}{l}\text { (Draghi et al 2006; Sahm } \\
\text { et al 2006; Jansen et al } \\
2007 \text { ) }\end{array}$} \\
\hline Vancomycin & $0.5-4$ & $1-2$ & 100 & \\
\hline Linezolid & $\leq 0.5->4$ & 2 & $99.4-99.8$ & \\
\hline Daptomycin & $0.12->1$ & $0.5-1$ & $99.6-99.7$ & \\
\hline \multicolumn{5}{|c|}{ Staphylococcus aureus: glycopeptide intermediate susceptible $(n=50)$} \\
\hline Telavancin & $0.125-1$ & 1 & NA & \multirow[t]{4}{*}{ (Leuthner et al 2006) } \\
\hline Vancomycin & $4-8$ & 8 & NR & \\
\hline Linezolid & $0.5-4$ & 2 & NR & \\
\hline Daptomycin & $0.25-2$ & I & NR & \\
\hline \multicolumn{5}{|c|}{ Streptococcus pneumoniae: penicillin susceptible $(n=37 I)$} \\
\hline Telavancin & $\leq 0.001-0.06$ & 0.03 & NA & \multirow{4}{*}{$\begin{array}{l}\text { (Draghi et al 2006; } \\
\text { Thornsberry et al 2006; } \\
\text { Jansen et al 2007) }\end{array}$} \\
\hline Vancomycin & $\leq 0.06-1$ & 0.5 & 100 & \\
\hline Linezolid & $0.25-2$ & $\mathrm{I}$ & 100 & \\
\hline Daptomycin & $\leq 0.03-1$ & 0.25 & NA & \\
\hline \multicolumn{5}{|c|}{ Streptococcus pneumoniae: penicillin resistant $(n=74)$} \\
\hline Telavancin & $0.008-0.03$ & $0.015-0.03$ & NA & \multirow{4}{*}{$\begin{array}{l}\text { (Draghi et al 2006; } \\
\text { Thornsberry et al 2006; } \\
\text { Jansen et al 2007) }\end{array}$} \\
\hline Vancomycin & $0.25-0.5$ & 0.5 & 100 & \\
\hline Linezolid & $0.5-1$ & 1 & 100 & \\
\hline Daptomycin & $0.06-0.5$ & $0.12-0.25$ & NA & \\
\hline \multicolumn{5}{|c|}{ Enterococcus faecalis: vancomycin susceptible $(n=928)$} \\
\hline Telavancin & $0.06-4$ & $0.5-1$ & NA & \multirow{4}{*}{$\begin{array}{l}\text { (Draghi et al } 2005 \text {; Sahm } \\
\text { et al 2006; Jansen et al } \\
2007 \text { ) }\end{array}$} \\
\hline Vancomycin & $\leq 0.5-4$ & 2 & 100 & \\
\hline Linezolid & $0.25-32$ & 2 & $99.8-100$ & \\
\hline Daptomycin & $\leq 0.015-4$ & $1-2$ & 100 & \\
\hline \multicolumn{5}{|c|}{ Enterococcus faecalis: vancomycin nonsusceptible $(n=60)$} \\
\hline Telavancin & $0.25-16$ & $8-16$ & NA & \multirow{4}{*}{$\begin{array}{l}\text { (Draghi et al 2006; Sahm } \\
\text { et al 2006; Jansen et al } \\
2007 \text { ) }\end{array}$} \\
\hline Vancomycin & $8->512$ & $>512$ & 0 & \\
\hline Linezolid & $0.5-2$ & $1-2$ & 100 & \\
\hline Daptomycin & $0.25-2$ & $\mathrm{I}-2$ & 100 & \\
\hline \multicolumn{5}{|c|}{ Enterococcus faecium: vancomycin susceptible $(n=427)$} \\
\hline Telavancin & $\leq 0.015-2$ & 0.25 & NA & \multirow{4}{*}{$\begin{array}{l}\text { (Draghi et al 2006; Sahm } \\
\text { et al 2006; Jansen et al } \\
2007 \text { ) }\end{array}$} \\
\hline Vancomycin & $\leq 0.5-4$ & 1 & 100 & \\
\hline Linezolid & $\leq 0.015-4$ & 2 & $97.8-100$ & \\
\hline Daptomycin & $\leq 0.015-8$ & 4 & $98.9-99.3$ & \\
\hline \multicolumn{5}{|c|}{ Enterococcus faecium: vancomycin nonsusceptible $(n=352)$} \\
\hline Telavancin & $\leq 0.015-16$ & $2-8$ & NA & \multirow{4}{*}{$\begin{array}{l}\text { (Draghi et al 2006; Sahm } \\
\text { et al 2006; Jansen et al } \\
2007 \text { ) }\end{array}$} \\
\hline Vancomycin & $8->512$ & $512->512$ & 0 & \\
\hline Linezolid & $1-16$ & 2 & $98.8-100$ & \\
\hline Daptomycin & $0.12-8$ & $2-4$ & $99.2-100$ & \\
\hline
\end{tabular}

${ }^{\mathrm{a}}$ Total number of isolates from all studies evaluated.

'Breakpoints based on Clinical and Laboratory Standards Institute guidelines (CSLI 2007)

Abbreviations: $\mathrm{MIC}_{90}$, minimum inhibitory concentration at which $90 \%$ of strains are inhibited; NA, not available for interpretation; NR, not reported.

telavancin interacts with the Gram-positive bacterial membrane to effect changes in membrane potential and permeability in a concentration-dependent manner (Higgins et al 2005). A strong correlation was observed between the bactericidal activity of telavancin and these membrane effects. Parallel control experiments using vancomycin in this experimental system showed no effect on membrane potential or cell viability at 60 minutes. Overall, this dual multimodal mechanism of action of telavancin thus appears to be responsible for its enhanced in vitro potency and rapid bactericidal activity against most Gram-positive bacteria (Laohavaleeson et al 2007).

\section{In vitro spectrum of activity}

Telavancin is active against both vancomycin-susceptible and some vancomycin-resistant Gram-positive organisms. MIC 
Table 2 Effect of dose on pharmacokinetic parameters of telavancin in healthy adult volunteers over 7 days

\begin{tabular}{|c|c|c|c|}
\hline \multirow[b]{2}{*}{ Pharmacokinetic parameters } & \multicolumn{3}{|c|}{ Dose } \\
\hline & $7.5 \mathrm{mg} / \mathrm{kg} / \mathrm{day}$ & $12.5 \mathrm{mg} / \mathrm{kg} /$ day & $15 \mathrm{mg} / \mathrm{kg} / \mathrm{day}$ \\
\hline Number of subjects & $\mathrm{n}=6$ & $n=6$ & $\mathrm{n}=4$ \\
\hline$C_{\text {max }}(\mu g / m L)$ & $96.7 \pm 19.8$ & $15 \mid \pm 17$ & $203 \pm 29$ \\
\hline $\mathrm{AUC}_{\mathrm{ss}}(\mu \mathrm{g} \cdot \mathrm{h} / \mathrm{mL})$ & $700 \pm 114$ & $1033 \pm 91$ & $1165 \pm 232$ \\
\hline$t_{1 / 2}(h)$ & $8.83 \pm 1.71$ & $9.11 \pm 2.33$ & $8.78 \pm 1.46$ \\
\hline $\mathrm{V}_{\mathrm{ss}}(\mathrm{mL} / \mathrm{kg})$ & $105 \pm 20$ & $119 \pm 18$ & $126 \pm 15$ \\
\hline $\mathrm{CL}(\mathrm{mL} / \mathrm{h} / \mathrm{kg})$ & $10.9 \pm 1.6$ & $12.2 \pm \mathrm{I} . \mathrm{I}$ & $13.3 \pm 2.6$ \\
\hline
\end{tabular}

Phase I study of pharmacokinetic data following 7-day treatment (Shaw et al 2005).

Values were reported as means \pm standard deviations.

Abbreviations: $A \cup C_{s s}$, area under curve at steady state; $\mathrm{CL}$, clearance; $\mathrm{C}_{\max }$, maximum serum concentration; $\mathrm{t}_{\mathrm{l} / 2}$, half-life; $\mathrm{V}_{\mathrm{ss}}$, volume of distribution at steady state.

values are elevated against VanA-type vancomycin-resistant enterococci. Like vancomycin, no activity is observed in vitro against Gram-negative bacteria. Breakpoints have not been established to date. Results from a European surveillance study showed that telavancin MICs range between 0.06 and $0.5 \mu \mathrm{g} / \mathrm{mL}$ for both MSSA and MRSA which was 2- to 4-fold lower than that for vancomycin, 4- to 80-fold lower than that for linezolid and 2-fold lower than that for daptomycin (Jansen et al 2007). Significantly, telavancin demonstrated excellent bactericidal activity against isolates of PVL-producing and non-PVL-producing CA-MRSA, with MIC and MBC values ranging from 0.25 to $1 \mu \mathrm{g} / \mathrm{ml}$ (Saravolatz et al 2007). Against 19 isolates of staphylococci not susceptible to daptomycin or linezolid, telavancin demonstrated MICs ranging from $0.06-1 \mu \mathrm{g} / \mathrm{ml}$ (Draghi 2005; Draghi et al 2006). Telavancin also displayed excellent activity against VISA derived from clinical isolates $\left(\mathrm{MIC}_{90}, 1 \mu \mathrm{g} / \mathrm{mL}\right.$ ), and was several times more potent than vancomycin $\left(\mathrm{MIC}_{90}, 8 \mu \mathrm{g} / \mathrm{mL}\right.$ ) (Leuthner et al 2006). Indeed, telavancin activity against VISA was comparable to or more potent than daptomycin and linezolid ( $\mathrm{MIC}_{90} \mathrm{~s} 1$ and $2 \mu \mathrm{g} / \mathrm{mL}$, respectively) (Leuthner et al 2006). In a time-kill study, the activity of telavancin was compared in 50 glycopeptide non-susceptible staphylococcal strains in the presence and absence of serum (Leuthner et al 2006). Included in this study were heteroresistant VISA, VISA, and VRSA strains. Telavancin demonstrated concentration dependent killing at or above 4 times the MIC of each respective strain. The telavancin MIC range for $\mathrm{VRSA}_{\mathrm{MI}}, \mathrm{VRSA}_{\mathrm{PA}}$, and $\mathrm{VRSA}_{\mathrm{NY}}$ was $1-4 \mu \mathrm{g} / \mathrm{mL}$ (Draghi et al 2005).

Although Staphylococcus epidermidis is a less common cause of clinical illness, it is important to note that the in vitro MIC values of telavancin are in the range of $0.03-0.5 \mu \mathrm{g} / \mathrm{mL}$ for methicillin-sensitive $S$. epidermidis and $0.25-1 \mu \mathrm{g} / \mathrm{mL}$ for methicillin-resistant S. epidermidis. Telavancin retains activity against teicoplanin and vancomycin-resistant $S$. epidermidis
(Draghi et al 2006; Leuthner et al 2006; Jansen et al 2007). This pathogen has assumed a greater role in line and surgical site infection in recent years.

Telavancin also has potent activity against species of streptococci, including PRSP with MICs in the $0.02-0.12 \mu \mathrm{g} / \mathrm{mL}$ range. MICs for telavancin vs streptococcus were lower than those for vancomycin, linezolid and daptomycin. MICs of telavancin for non-pneumococcal strains of Streptococcus were reported as $0.015-0.12 \mu \mathrm{g} / \mathrm{mL}$ (Jansen et al 2007).

Telavancin has good activity against most strains of Gram-positive anaerobes found in cSSSIs like Clostridium jeikeium. Likewise, the $\mathrm{MIC}_{90}$ values for telavancin, vancomycin, daptomycin and linezolid against $C$. difficile, an important super-infection resulting from antibiotic therapy, were $0.25,1,2$, and 8 , respectively (Goldstein et al 2004; Finegold et al 2005).

\section{Pharmacokinetics}

In preclinical studies, pharmacokinetic parameters of telavancin were evaluated in mice, rats, dogs and monkeys. Tissue distribution was studied in rats and dogs; an in vitro assay of microsomal/P450 mediated metabolism was performed, and drug - drug interactions were studied in rats. Preliminary identification of metabolites was also determined. These studies demonstrated linear kinetics and a modest increase in $T_{1 / 2}$ after prolonged dosing at 13 weeks only in the rat. Urinary excretion was the primary route of elimination with little or no metabolism (Shaw et al 2005).

Most data on the pharmacokinetic profile of telavancin have been collected in Phase I studies involving 200 volunteers, including 16 healthy elderly subjects (Duchin 2004), 16 subjects with moderate hepatic impairment (Wong et al 2006) and 22 with varying degrees of renal impairment (Duchin et al 2004). The data provided recommendation that the therapeutic telavancin dosage in patients with a creatinine clearance above $50 \mathrm{~mL} / \mathrm{min}$ is $10 \mathrm{mg} / \mathrm{kg} /$ day. 
The observed half-life of telavancin at this dosage was 7-9 hours; plasma concentrations increased in a linear fashion in proportion to dose without evidence of clinically significant drug accumulation (Shaw et al 2005). These data further support the recommendation for once daily dosing. In a study investigating the effect of renal impairment on the pharmacokinetics of a single dose of telavancin (Duchin et al 2004), a 2- to 3-fold increase in exposure to telavancin was found. Thus, for patients with creatinine clearance in the range of $30-50 \mathrm{~mL} / \mathrm{min}$, a $75 \%$ dose $(7.5 \mathrm{mg} / \mathrm{kg})$ is recommended, and in patients with end-stage renal disease and those with a creatinine clearance $<30 \mathrm{~mL} / \mathrm{min}$, the dosing interval should be extended to every 48 hours (Duchin et al 2004). In vitro studies have been performed in a model of continuous venovenous hemofiltration and continuous venovenous hemodialysis, but no recommendations for dosing in renal failure patients undergoing dialysis were proposed (Patel et al 2006a, b). The primary hepatic metabolite identified was a hydroxylated form of the parent compound. In a study investigating the effect of moderate hepatic impairment on the pharmacokinetics of a single dose of telavancin $(10 \mathrm{mg} / \mathrm{kg})$, no meaningful differences on pharmacokinetics were observed between normal subjects and subjects with moderate hepatic impairment (Wong et al 2006). In a study of pharmacokinetics in subjects 65 years and older (Duchin et al 2004), no effect of age was found. In laboratory analyses, it was found that telavancin could interfere with coagulation assays and with dye tests for urinary protein (Laohavaleeson et al 2007).

The concentration of telavancin in tissues that are common sites of infection was studied extensively. The steady-state AUC in blister fluid was $40 \%$ of that found in plasma but was at a level sufficient to eradicate pathogens which might be present (Sun et al 2006). In epithelial lining fluid (ELF), concentrations of telavancin were $10 \%$ of that in plasma, but levels were 2-8 times higher than the MIC of telavancin versus MRSA (Wong et al 2007). In alveolar macrophages, levels of telavancin were reported to be higher than in ELF and further, in contrast to daptomycin, did not seem to be affected by the presence of lung surfactant (Wong et al 2007).

\section{Pharmacodynamics}

Telavancin pharmacodynamic studies have been conducted in several in vitro studies and in vivo animal models of infection. The dynamic and static antimicrobial effects of telavancin against MRSA and MSSA have been simulated in an in vitro kinetic model (Odenholt et al 2007). At a clinically feasible area under the curve/MIC ratio of 50, telavancin produced $>3-\log _{10}$ killing at $6-8 \mathrm{~h}$ postexposure in the absence and presence of $50 \% \mathrm{w} / \mathrm{v}$ human plasma. In this study, telavancin was the only antibacterial to kill MRSA and MSSA in their nongrowing phase (Odenholt et al 2007). In the mouse neutropenic thigh (MNT) model, the 24-hour AUC/MIC ratio was identified as the best predictor of efficacy (Hegde et al 2004). Concentration- dependent activity was demonstrated against a variety of Gram-positive bacteria, including MSSA, MRSA, PSSP, PRSP, and vancomycin-resistant Enterococcusfaecalis. In studies of intravenously administered telavancin, the effective dose for $50 \%$ bactericidal activity $\left(\mathrm{ED}_{50}\right)$ was estimated to range from 0.5 to $6.6 \mathrm{mg} / \mathrm{kg}$ with pretreatment titers reduced by $3 \mathrm{log} \mathrm{CFU} / \mathrm{g}$, supporting the bactericidal properties of this molecule. Telavancin, vancomycin, and linezolid were also efficacious and more potent against MRSA in the murine subcutaneous infection model (immune system intact) compared with the MNT model. This increase in potency was, however, disproportionately greater for vancomycin and linezolid when compared to telavancin, suggesting that the activity of telavancin is less dependent on a competent immune system. In a model of MRSA pneumonia, established in immunocompromised mice, intravenous or subcutaneously dosed telavancin demonstrated superior efficacy to intravenous vancomycin and linezolid (Reyes et al 2005).

In a model of bacteremia caused by MRSA in immunocompromised mice, telavancin was more efficacious than vancomycin in reducing blood bacterial titers and improving survival (Reyes et al 2006). Telavancin was also efficacious in a rabbit model of aortic valve endocarditis evoked by MRSA and VISA (Madrigal et al 2005).

\section{Clinical studies}

Two phase II clinical trials were conducted, the FAST and FAST 2 studies. Both studies were randomized, doubleblind trials involving centers in the United States and South Africa (Stryjewski et al 2005; Stryjewski et al 2006). Both studies were conducted to assess safety and explore efficacy of telavancin in patients with cSSSI. In FAST, a total of 167 adult patients received either $7.5 \mathrm{mg} / \mathrm{kg}$ of telavancin intravenously every 24 hours or the standard therapies ( $1 \mathrm{~g}$ vancomycin every 12 hours, $0.5-1 \mathrm{~g}$ cloxacillin every 6 hours, or $2 \mathrm{~g}$ nafcillin - oxacillin every 6 hours) (Stryjewski et al 2005). Dose adjustment of vancomycin was permitted based on site-specific guidelines. 84 patients were randomized to receive telavancin, while 83 patients received the standard therapies. Based upon pre-randomization 
choice of the investigator, $75 \%$ of those receiving standard therapy were given vancomycin, no doubt a reflection of increased awareness of high prevalence rates of MRSA. Drug was given for 7-14 days based on clinical response and the investigators judgment. $S$. aureus was isolated at baseline in approximately 50 patients in each treatment group, and MRSA was identified in approximately 50\% of the $S$. aureus isolates. Overall cure rates were similar at $79 \%$ and $80 \%$ for telavancin and the standard therapy group respectively $(p=0.53)$. In cases with MRSA, cure rates were $82 \%$ and $69 \%$ for telavancin and the standard therapy group, respectively. However, this difference was not statistically significant due to the relatively small number of patients involved. In microbiologically evaluable patients inclusive of all pathogens, clinical cure rates were $80 \%$ and $82 \%$ for telavancin and the standard therapy group respectively $(\mathrm{p}=0.83)$.

In FAST 2, the primary goal was to compare the efficacy of telavancin dosed at $10 \mathrm{mg} / \mathrm{kg}$ intravenously every 24 hours with standard therapies (same as in FAST) (Stryjewski et al 2006). A total of 195 patients were enrolled of which 100 received telavancin. In this study, 93\% of the standard therapy patients received vancomycin. Among the 91 microbiologically evaluable patients with $S$. aureus at baseline, cure rates were $96 \%$ and $90 \%$ for telavancin and the standard therapy group respectively. In the 45 microbiologically evaluable patients with MRSA at baseline, cure rates were also $96 \%$ and $90 \%$ for telavancin and the standard therapy group respectively. In terms of microbiologic eradication, telavancin trended towards a better outcome in patients with S. aureus than standard therapy ( $92 \%$ vs $78 \%$, $\mathrm{p}=0.07)$, but had a statistically significant better eradication rates in patients with MRSA $(92 \%$ versus $68 \%, \mathrm{p}=0.04)$ (Stryjewski et al 2006; Drew 2007).

Two large phase III clinical trials, ATLAS I and ATLAS II, were completed in mid 2006 comparing telavancin safety and efficacy to that of vancomycin in patients with cSSSI primarily due to MRSA (Corey et al 2006). These studies were identical in design and were multinational, randomized, and double-blind. Patients were enrolled from 129 sites in 21 countries. Both studies met the primary efficacy endpoint of non-inferiority to vancomycin in clinical cure rate at the follow-up (test of cure) visit, which occurred 7-14 days after the last dose of study medication.

Results from the ATLAS studies have not yet been fully published; however preliminary reports state that patients were enrolled from 129 sites in 21 countries. Both studies met the primary efficacy endpoint of non-inferiority to vancomycin in clinical cure rate at the follow-up (test of cure) visit, which occurred 7-14 days after the last dose of study medication. Telavancin-treated patients with cSSSI caused by MRSA trended toward an overall better cure rate than vancomycin (90.6\% versus $86.4 \%$ ) which was not statistically significant (difference $4.1 \%, 95 \% \mathrm{CI},-1.1,9.3$ ). In addition, telavancin showed a higher eradication rate of MRSA than vancomycin (89.9\% versus $85.4 \%$ ), which again was not statistically significant (Difference 4.4\%, 95\%CI, -0.9\%, 9.8\%) (Corey, Stryjewski et al 12-15 October 2006).

Presently, phase III clinical studies are ongoing investigating telavancin in hospital-acquired pneumonia caused by Gram-positive bacteria.

\section{Safety and tolerability}

Telavancin has generally been well-tolerated in Phase I studies of healthy volunteers and in clinical studies involving patients with cSSSI (Shaw et al 2005; Corey et al 2006; Stryjewski et al 2006). In the phase III ATLAS studies, the incidence of adverse events leading to discontinuation of treatment was $8 \%$ in the telavancin group $(n=928)$ and $6 \%$ in the vancomycin group $(n=939)$ (Corey et al 2006). The most common treatment-emergent adverse events associated with telavancin were taste alterations (33\%), nausea (27\%), headache and vomiting (both 14\%), foamy urine (13\%), and insomnia and constipation (both 10\%) (Corey et al 2006). In the FAST 2 study, increases in serum creatinine from normal to abnormal were more frequent in patients receiving telavancin than vancomycin (5 of 100 patients vs 0 of 95 patients) (Stryjewski et al 2006). Maximum concentrations of serum creatinine were $\leq 1.8 \mathrm{mg} / \mathrm{dL}$ in all but one telavancin patient, and creatinine values returned to the normal range for this patient during follow-up. In a separate safety study, at higher doses, some vomiting and the infusion-related reaction previously described with vancomycin therapy, known as "red-man syndrome", occurred in a small percentage of telavancin recipients (Barriere et al 2004). A clinically insignificant prolongation of the QTc was noted in healthy volunteers $(<5 \mathrm{msec})$ and did not appear to be dose related (Barriere et al 2004). In particular, no telavancin recipients in this study had a prolonged prolonged QTcF of $>500 \mathrm{msec}$ or any abnormal U-waves (Barriere et al 2004). In ATLAS (Corey et al 2006), electrocardiogram analyses identified a prolonged QTcF of $>500 \mathrm{msec}$ in 1 telavancin patient and 2 vancomycin patients and prolonged QTc interval by $>60 \mathrm{msec}$ in 10 telavancin patients $(1 \%)$ and 5 vancomycin patients $(0.5 \%)$. None of these ECG abnormalities were associated with cardiac events. 


\section{Conclusions}

Studies to date have shown that telavancin, a new lipoglycopeptide antibiotic that is a semi-synthetic derivative of vancomycin, has rapid bactericidal activity against a variety of clinically important Gram-positive pathogens known to be the predominant causative bacteria in cSSSIs. Overall outcome in studies using extended spectrum penicillins or vancomycin as the comparator agents versus telavancin have demonstrated comparable clinical outcomes. In the subset of patients infected with MRSA, results in a phase II study have shown a statistically significant better outcome in the telavancin treated patients (Laohavaleeson et al 2007). There was a similar trend, although not statistically significant in the phase III trials. In vitro studies have demonstrated excellent activity against multi-drug resistant strains including MRSA, PRSP, heteroresistant VISA, VISA, and VRSA (Laohavaleeson et al 2007). Data from animal models of infection support the bactericidal properties of telavancin. Adverse events associated with telavancin treatment have been largely mild to moderate in severity, and except for nausea, vomiting, taste disturbance, and foamy urine, occurred at similar frequencies to that of comparator drugs in phase II and III studies. Laboratory abnormalities of significance include increased serum creatinine (largely low-grade), occurring in a small proportion of patients. Serum creatinine returned to baseline following discontinuation of drug (Stryjewski et al 2006). The work referenced in this summary of telavancin supports its safety and effectiveness in the treatment of cSSSIs. With FDA approval and that of worldwide regulatory agencies, it will provide an important new option in the treatment of serious infections caused by Gram-positive bacteria, especially those bacteria resistant to many of the currently available agents.

\section{Expert opinion}

Although the problem of resistant strains of Gram-positive organisms was initially largely confined to the in-hospital setting, the eruption of CA-MRSA strains and the increasing prevalence of strains heteroresistant to vancomycin have led to considerable concern in the management of serious infections likely to be caused by these pathogens. The virulence of the community-acquired strain has resulted in an unprecedented number of patients appearing in emergency departments across the United States with large abscesses surrounded by extensive cellulitic changes, frequently in the setting of no known disruption in the integrity of the skin. Hence the well known "must have been a spider bite" complaint has emerged as a risk factor for CA-MRSA (Moran et al 2006).
Clindamycin, trimethoprim-sulfamethoxazole, and the tetracyclines are first-line therapies for use in the treatment of these infections, especially in the outpatient setting. However, there are no controlled clinical trials to support their efficacy and it is unlikely that such studies will be done since these agents are all now generic drugs. Unlike cSSSIs caused by CA-MRSA, those by HA-MRSA are often recalcitrant. As the distinction between cSSSIs due to CA-MRSA and HA-MRSA becomes blurred, it is increasingly difficult for clinicians to rely on traditional agents as empirical therapy. Given that the development of intermediate and frank resistant strains of $S$. aureus to vancomycin has led to clinical failures, it is imperative that more effective agents become available. In this regard, telavancin shows promise as an excellent choice for the management of cSSSI though its place relative to traditional and developmental agents will ultimately be decided by results from ongoing preclinical, clinical and economic studies. Each of the agents currently in development for cSSSI are associated with specific advantages and limitations relative to vancomycin. For example, the long elimination half-life of dalbavancin appears to offer the opportunity for a once-weekly dosing strategy on an outpatient basis, but this pharmacokinetic may also be a problem since any dalbavancin-associated adverse effects are likely to be enduring. The development of ceftobirole and iclaprim is welcomed, as these agents are derived from parent molecules with a traditionally good safety profile; however, both agents possess a solitary mechanism of action that may increase the chance of rapid resistance development if used empirically.

As referenced in the body of this paper, telavancin clinical efficacy, microbiological eradication, and safety have been demonstrated for a variety of Gram-positive infections, including those caused by resistant strains of $S$. aureus and S. pneumonia. Its multifunctional, cidal mode of action confers an extremely low potential for resistance development while its once-daily dosing regimen lends itself to use in the outpatient setting. In light of the added expense of in-hospital care and the pressure to limit the duration of hospital stays, an economic study has been established to determine the exact cost savings relative to vancomycin. Release of the PVL toxin is a major concern in serious necrotizing infections. Important studies are currently investigating the bactericidal activity of telavancin. If the findings determine that telavancin does not induce prokaryotic cell lysis, then by inference, telavancin therapy may minimize release of preformed PVL toxin. Its rapidly bactericidal activity likewise may possibly further reduce production of this toxin. No doubt this information will add to the clinician's comfort in use of this agent in serious infections. 


\section{References}

Barriere SF, Genter F, et al. 2004. Effects of a new antibacterial, telavancin, on cardiac repolarization (QTc interval duration) in healthy subjects. $J$ Clin Pharmacol, 44:689-95.

Beauregard DA, Williams DH, et al. 1995. Dimerization and membrane anchors in extracellular targeting of vancomycin group antibiotics. Antimicrob Agents Chemother, 39:781-5.

CDC. 2002a. Staphylococcus aureus resistant to vancomycin - United States, 2002. MMWR Morb Mortal Wkly Rep, 51:565-7.

CDC. 2002b. Vancomycin-resistant Staphylococcus aureus - Pennsylvania, 2002. MMWR Morb Mortal Wkly Rep, 51:902.

Chambers HF. 2001. The changing epidemiology of Staphylococcus aureus? Emerg Infect Dis, 7:78-82.

[CLSI] Clinical and Laboratory Standards Institute. 2007. Performance Standards for Antimicrobial Susceptibility Testing; Seventeenth Informational Supplement M100-S17. Volume, DOI:

Corey R, Stryjewski M, et al. 2006. Telavancin for the treatment of complicated skin and skin structure infections (cSSSI): results of the ATLAS I study. 44th Infectious Disease Society of America. Toronto, Canada.

Davis SL, Perri MB, et al. 2007. Epidemiology and outcomes of communityassociated methicillin-resistant Staphylococcus aureus infection. J Clin Microbiol, 45:1705-11.

Draghi D, Jones M, Thornsberry C, et al. 2005. Telavancin activity against current and diverse Staphylococcus aureus populations. 45th Interscience Conference on Antimicrobial Agents and Cemotherapy. Washington D.C., USA.

Draghi DC, Benton BM, et al. 2006. Baseline antistaphylococcal profile of telavancin: Results of the 2004-2005 US Surveillance Initiative. 46th Annual ICAAC. San Francisco, CA.

Drew RH. 2007. Emerging options for treatment of invasive, multidrugresistant Staphylococcus aureus infections. Pharmacotherapy, 27:227-49.

Duchin K, Shaw J, et al. 2004. Single dose pharmacokinetics (PK) of telavancin (TLV) in healthy elderly subjects. 14th European Congress of Clinical Microbiology and Infectious Diseases. Prague, Czech Republic.

Finegold SM, Song Y, et al. 2005. Clostridium clostridioforme: a mixture of three clinically important species. Eur J Clin Microbiol Infect Dis, 24:319-24.

Goldstein EJ, Citron DM, et al. 2004. In vitro activities of the new semisynthetic glycopeptide telavancin (TD-6424), vancomycin, daptomycin, linezolid, and four comparator agents against anaerobic gram-positive species and Corynebacterium spp. Antimicrob Agents Chemother, 48:2149-52.

Goossens H. 2006. Antibiotic resistance: Highlights of the 16th European Congress on Clinical Microbiology and Infectious Diseases. 16th European Congress on Clinical Microbiology and Infectious Diseases, Nice, France.

Hegde SS, Reyes N, et al. 2004. Pharmacodynamics of telavancin (TD6424), a novel bactericidal agent, against gram-positive bacteria. Antimicrob Agents Chemother, 48:3043-50.

Hershow RC, Khayr WF, et al. 1998. Ciprofloxacin resistance in methicillinresistant Staphylococcus aureus: associated factors and resistance to other antibiotics. Am J Ther, 5:213-20.

Higgins DL, Chang R, et al. 2005. Telavancin, a multifunctional lipoglycopeptide, disrupts both cell wall synthesis and cell membrane integrity in methicillin-resistant Staphylococcus aureus. Antimicrob Agents Chemother, 49:1127-34

Hiramatsu K, Hanaki H, et al. 1997. Methicillin-resistant Staphylococcus aureus clinical strain with reduced vancomycin susceptibility. $J$ Antimicrob Chemother, 40:135-6.

Hooper DC. 1999. Mechanisms of fluoroquinolone resistance. Drug Resist Updat, 2:38-55.

Jansen WT, Verel A, et al. 2007. In vitro activity of telavancin against gram-positive clinical isolates recently obtained in Europe. Antimicrob Agents Chemother, 51:3420-4.

Jevons M. 1961. Celbenin-resistant staphylococci. BMJ, 1:124-5.
Johnson AP, Aucken HM, et al. 2001. Dominance of EMRSA-15 and -16 among MRSA causing nosocomial bacteraemia in the UK: analysis of isolates from the European Antimicrobial Resistance Surveillance System (EARSS) J Antimicrob Chemother, 48:143-4.

Judice JK, Pace JL. 2003. Semi-synthetic glycopeptide antibacterials. Bioorg MedChem Lett, 13:4165-8.

Laohavaleeson S, Kuti JL, et al. 2007. Telavancin: a novel lipoglycopeptide for serious gram-positive infections. Expert Opin Investig Drugs, 16:347-57.

Leadbetter MR, Adams SM, et al. 2004. Hydrophobic vancomycin derivatives with improved ADME properties: discovery of telavancin (TD6424). J Antibiot (Tokyo), 57:326-36.

Leuthner KD, Cheung CM, et al. 2006. Comparative activity of the new lipoglycopeptide telavancin in the presence and absence of serum against 50 glycopeptide non-susceptible staphylococci and three vancomycin-resistant Staphylococcus aureus. J Antimicrob Chemother, $58: 338-43$.

Levine DP. 2006. Vancomycin: a history. Clin Infect Dis, 42(Suppl 1): S5-12.

Madrigal AG, Basuino L, et al. 2005. Efficacy of telavancin in a rabbit model of aortic valve endocarditis due to methicillin-resistant Staphylococcus aureus or vancomycin-intermediate Staphylococcus aureus. Antimicrob Agents Chemother, 49 3163-5.

Maor Y, Rahav G, et al. 2007. Prevalence and characteristics of heteroresistant vancomycin-intermediate Staphylococcus aureus bacteremia in a tertiary care center. J Clin Microbiol, 45:1511-4.

Moellering RC Jr. 2006. Vancomycin: a 50-year reassessment. Clin Infect Dis, 42(Suppl 1):S3-4.

Moran GJ, Krishnadasan A, et al. 2006. Methicillin-resistant S. aureus infections among patients in the emergency department. $N$ Engl $J$ Med, 355:666-74.

Nishimura S, Tsurumoto T, et al. 2006. Antimicrobial susceptibility of Staphylococcus aureus and Staphylococcus epidermidis biofilms isolated from infected total hip arthroplasty cases. J Orthop Sci, 11:46-50.

Odenholt I, Lowdin E, et al. 2007. Pharmacodynamic effects of telavancin against methicillin-resistant and methicillin-susceptible Staphylococcus aureus strains in the presence of human albumin or serum and in an in vitro kinetic model. Antimicrob Agents Chemother, 51:3311-6.

Patel J, Grio M, et al. 2006a. Telavancin pharmacokinetics during in vitro continuous venovenous hemodialysis. 16th European Congress of Clinical Microbiology and Infectious Diseases. Nice, France. 1539.

Patel J, Grio M, et al. 2006b. Telavancin transmembrane clearance during in vitro continuous venovenous hemofiltration. 16th European Congress of Clinical Microbiology and Infectious Diseases. Nice, France. 1538.

Pfizer. 2007. "http://www.pfizer.com." Accessed 23 June 2007 URL: http://www.pfizer.com.

Reyes N, Skinner R, et al. 2006. Efficacy of telavancin in a murine model of bacteraemia induced by methicillin-resistant Staphylococcus aureus. $J$ Antimicrob Chemother, 58:462-5.

Reyes N, Skinner R, et al. 2005. Efficacy of telavancin (TD-6424), a rapidly bactericidal lipoglycopeptide with multiple mechanisms of action, in a murine model of pneumonia induced by methicillin-resistant Staphylococcus aureus. Antimicrob Agents Chemother, 49:4344-6.

Rice LB. 2006. Antimicrobial resistance in gram-positive bacteria. Am J Med, 119(Suppl 1):S11-9; discussion S62-70.

Sakoulas G, Moellering RC Jr, et al. 2006. Adaptation of methicillin-resistant Staphylococcus aureus in the face of vancomycin therapy. Clin Infect Dis, 42(Suppl 1):S40-50.

Saravolatz LD, Pawlak J, et al. 2007. Comparative activity of telavancin against isolates of community-associated methicillin-resistant Staphylococcus aureus. J Antimicrob Chemother, 60:406-9.

Shaw JP, Seroogy J, et al. 2005. Pharmacokinetics, serum inhibitory and bactericidal activity, and safety of telavancin in healthy subjects. Antimicrob Agents Chemother, 49:195-201.

Spink W, Ferris V. 1945. Quantitative action of penicillin inhibitor from penicillin-resistant strains in staphylococci. Science, 102:221-3. 
Stevens DL. 2006. The role of vancomycin in the treatment paradigm. Clin Infect Dis, 42(Suppl 1):S51-7.

Stryjewski ME, Chu VH, et al. 2006. Telavancin versus standard therapy for treatment of complicated skin and skin structure infections caused by gram-positive bacteria: FAST 2 study. Antimicrob Agents Chemother, 50:862-7.

Stryjewski ME, O'Riordan WD, et al. 2005. Telavancin versus standard therapy for treatment of complicated skin and soft-tissue infections due to gram-positive bacteria. Clin Infect Dis, 40:1601-7.

Styers D, Sheehan DJ, et al. 2006. Laboratory-based surveillance of current antimicrobial resistance patterns and trends among Staphylococcus aureus: 2005 status in the United States. Ann Clin Microbiol Antimicrob, 5:2.

Sun HK, Duchin K, et al. 2006. Tissue penetration of telavancin after intravenous administration in healthy subjects. Antimicrob Agents Chemother, 50:788-90.
Swartz MN. 1994. Hospital-acquired infections: diseases with increasingly limited therapies. Proc Natl Acad Sci USA, 91:2420-7.

Talbot GH, Bradley J, et al. 2006. Bad bugs need drugs: an update on the development pipeline from the Antimicrobial Availability Task Force of the Infectious Diseases Society of America. Clin Infect Dis, 42:657-68.

Van Bambeke F. 2004. Glycopeptides in clinical development: pharmacological profile and clinical perspectives. Curr Opin Pharmacol, 4:471-8.

Wong S, Shaw J, et al. 2007. Penetration of telavancin into pulmonary epithelial lining fluid and alveolar macrophages. 17th European Congress of Clinical Microbiology and Infectious Diseases. ICC, Munich, Germany.

Wong S, Shaw JP, et al. 2006. Pharmacokinetics of intravenous telavancin in subjects with hepatic impairment. 2006 ICAAC Meeting. San Francisco, CA. 